\title{
Has the Universe always expanded?
}

\author{
Patrick Peter ${ }^{1}$ and Nelson Pinto-Neto ${ }^{2}$ \\ ${ }^{1}$ Institut d'Astrophysique de Paris, 98bis boulevard Arago, 75014 Paris, France. \\ ${ }^{2}$ Centro Brasileiro de Pesquisas Físicas, Rue Dr. Xavier Sigaud 150, Urca 22290-180 - Rio de Janeiro, RJ, Brazil
}

(11 September 2001)

\begin{abstract}
We consider a cosmological setting for which the currently expanding era is preceded by a contracting phase, that is, we assume the Universe experienced at least one bounce. We show that scalar hydrodynamic perturbations lead to a singular behavior of the Bardeen potential and/or its derivatives (i.e. the curvature) for whatever Universe model for which the last bounce epoch can be smoothly and causally joined to the radiation dominated era. Such a Universe would be filled with non-linear perturbations long before nucleosynthesis, and would thus be incompatible with observations. We therefore conclude that no observable bounce could possibly have taken place in the early universe if Einstein gravity together with hydrodynamical fluids is to describe its evolution, and hence, under these conditions, that the Universe has always expanded.
\end{abstract}

98.80.Hw, 98.80.Cq

\section{INTRODUCTION}

Since the development of singularity theorems [1], it has been a paradigm that the Standard Big Bang (SBB) cosmology [2] requires the Universe to have emerged from an initial singularity. However, as all theorems, those concerning singularities were based on a set of hypothesis. In the 1970's and after, many non-singular cosmological models with bounces were constructed [3] 3 where indeed one or more of the hypothesis of the theorems were violated, such as, for instance, energy conditions or the validity of Einstein gravity.

More recently, superstring-inspired models suggested that this singularity might have been avoided if gravity was modified somehow in the near Planckian regime for which a maximal curvature was supposed to be reachable. Note that although this model seems to have always expanded in the Jordan's frame, a bounce behavior is also present in the Einstein frame. Such a pre Big Bang (PBB) scenario [5] is, however, plagued with various problem, as, e.g. the graceful exit [6], and fails to reproduce the primordial perturbation spectrum as observed in the recent Cosmic Microwave Background $(\mathrm{CMB})$ data [7]. The non-singular hypothesis was revigorated even more recently with the Ekpyrotic Universe proposal [8].

Quantum gravity also provides new ways out of the singularity theorems. For instance, models exist in which an expanding Universe region might originate from a Black Hole collapse [9]. Quantum cosmology, by the Wheelerde Witt equation, also yields bouncing behaviors for the scale factor [10,11]. However, in this case, whether the Universe actually avoids the singularity may depend on the interpretation that is given to its wave function. In

\footnotetext{
*Already in the 1930's, a non-singular cosmological model

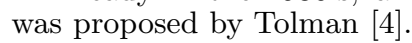

particular, in the many-world view [12], it is the expectation value of the scale factor that bounces, so that, in practice, nothing can be said as to the value it takes in the realization corresponding to our Universe. However, in the ontological interpretation [13] of the wave function of the Universe, the Bohmian trajectory, being meaningful, implies an actual physical bounce. (Note the interesting fact that this interpretation also offers a natural solution 114 to the graceful exit problem in the PBB scenario.)

In this paper, we restrict our attention to pure general relativity (GR) and examine a Friedman-RobertsonWalker (FRW) setting whose stress-energy source comes from a collection of perfect fluids (having vanishing anisotropic stresses). We then assume that a bounce took place at some instant in time and concentrate on observationally meaningful models, namely those for which the bounce epoch can be smoothly and causally connected to a radiation dominated phase where Big Bang Nucleosynthesis (BBN), assumed as a physical requirement in whatever model, can safely take place. Such Universes will be called "realistic" hereafter. What precedes the bounce itself is also left out of our analysis which is thus not restricted to non-singular models. In short, we just assume that, for some reason, our Universe has not always expanded, and we question the observable consequences of this possibility.

This work is to be contrasted with Ref. [15] in which the mathematical conditions on the total fluid for the bounce to occur are derived (see the Appendix for a physical restriction of these conditions) : our analysis is in some sense a follow-up of this work as we examine stability.

In the framework defined above, we investigate the behavior of scalar adiabatic perturbations near the bounce through the Bardeen gauge-invariant potential. We show that these perturbations can grow without limit in two situations. One, when the Universe passes through the bounce itself. The other, if the existence of the bounce 
imposes a violation of the Null Energy Condition (NEC) (and hence of all the other energy conditions [15]), when the Universe makes the transition from the region where the NEC is violated to the region where it is not 1 . In all cases of physical interest, at least one of these instabilities occurs. These facts, in turn, imply that there must exist a point in time where, apart for infinitely fine-tuned initial conditions (and yet for all wavenumbers at the same time), the perturbations grow unbounded. This means that if such a bounce had occurred, then very large inhomogeneities, at all scales, would have been formed very early on, that would presumably have turn non linear very rapidly, i.e. long before nucleosynthesis. Such a conclusion is in flagrant contradiction with BBN, so that we are led to postulate a no-bounce conjecture [18].

In fact, our conclusion is more general than that. Even without bounce, the point (in time) where the NEC is recovered (assuming it has been violated in the past, which is the case for the majority of bouncing Universes) yields divergences in the primordial perturbations. As a result, the PBB models, which may have no bounce, acquires a new constraint as follows : the graceful exit problem, in order to be solved, also requires, in many instances [19], a violation of the NEC for a finite amount of time (see however Ref. [14]|). Models should then take into account the fact that the recovery of the NEC should not occur at a time where our hypothesis (GR plus hydrodynamical perturbations) are already valid to avoid the excess perturbation problem.

Finally, there is the special case, which is actually not a bounce, for which the scale factor, although never decreasing in time, happens to stop at an instant $\left(a\left(\eta_{0}\right)=\right.$ $a_{0}+b \eta_{0}^{2 i+1}+\ldots$ for some $\eta_{0}$, with $i \geq 1$ ). Such a "pseudobounce" situation is included in our analysis, and it also generates a cosmological catastrophe. One must therefore conclude that the expansion must be strictly monotonous in order to make sense.

In what follows, after a short review (Sec. III) on the essentials of cosmological perturbation theory in the framework with which we are concerned, heavily based on Ref. 20], we detail the structure of a bouncing epoch (Sec. III) by making an expansion of the scale factor in powers of the conformal time. We then go on to evaluate the evolution of the primordial perturbations (in a gauge invariant manner in order to avoid spurious modes) around the bounce in Sec. IV, establishing our results. Secs. V and VI are devoted to discussions and conclusions, while a final appendix shows that connecting the bouncing phase with the observable Universe yields constraints on the parameters appearing in the expansion

\footnotetext{
${ }^{\dagger}$ Fluids which violate the NEC are called exotic matter. They have strange properties but there is no theorem asserting their impossibility on macroscopic scales [16] (one way or the other) For examples of energy-momentum tensors where the NEC is violated, see Ref. 17]
}

and exhibits clearly the violation of the NEC in many cases.

\section{CLASSICAL COSMOLOGICAL PERTURBATION THEORY AND THE NULL ENERGY CONDITION}

The purpose of this section is to recall various definitions and evolution equations for scalar hydrodynamic perturbation, for the sake of self-consistency.

Cosmological perturbations are expected to be produced quantum mechanically during the early stage of the evolution of the Universe. Expansion drives the quantum mechanical fluctuations of any field present in one's favorite model to classical values, which then evolve according to GR. Although we will concentrate on the subsequent evolution of primordial perturbations as soon as they have been produced, we shall also summarize the actual process of building them as the quantum modes of the field. This quantum analysis yield different insights into the nature of the problem.

The background metric we shall be using is

$$
\mathrm{d} s^{2}=a^{2}(\eta)\left(\mathrm{d} \eta^{2}-\gamma_{i j} \mathrm{~d} x^{i} \mathrm{~d} x^{j}\right),
$$

with $\eta$ the conformal time, related to the cosmic time $t$ through the scale factor $a, a \mathrm{~d} \eta=\mathrm{d} t$, and

$$
\gamma_{i j} \equiv \frac{\delta_{i j}}{\left[1+\frac{1}{4} \mathcal{K} \mathbf{x}\right]^{2}}
$$

the 3 -space metric. Here, $\mathcal{K}=0, \pm 1$ is the sign of the curvature. The general form of metric perturbations on this background reads

$$
\begin{aligned}
\mathrm{d} s^{2}= & a^{2}(\eta)\left\{(1+2 \phi) \mathrm{d} \eta^{2}-2 B_{; i} \mathrm{~d} \eta \mathrm{d} x^{i}\right. \\
& \left.-\left[(1-2 \psi) \gamma_{i j}+2 E_{; i j}\right] \mathrm{d} x^{i} \mathrm{~d} x^{j}\right\} .
\end{aligned}
$$

From this form, it is convenient to define the gaugeinvariant Bardeen potentials [21]

$$
\begin{gathered}
\Phi=\phi+\frac{1}{a}\left[\left(B-E^{\prime}\right) a\right]^{\prime}, \\
\Psi=\psi-\frac{a^{\prime}}{a}\left(B-E^{\prime}\right),
\end{gathered}
$$

(where a prime means a derivative with respect to conformal time), in terms of which it is possible to expand the perturbed Einstein equation in a gauge independent way. The latter are further simplified in the particular case of vanishing anisotropic stress for which $\Psi=\Phi$. In what follows, we shall restrict our attention to this special case. Note that for perturbation theory to be valid, and space not to become very inhomogeneous, the requirement $|\Phi| \ll 1$ should hold at all times and at all wavelengths of perturbation. The stress energy tensor, source of Einstein field equations, will take the form 


$$
T_{\nu}^{\mu}=(\epsilon+p) u^{\mu} u_{\nu}-p \delta_{\nu}^{\mu},
$$

for energy density $\epsilon$, pressure $p$ and 4 -velocity $\mathbf{u}$. Following Ref. [1], one then defines the Null Energy Condition through the Null Convergence Condition which requires the Ricci tensor to satisfy $R_{\mu \nu} n^{\mu} n^{\nu} \geq 0$ for all null vector $n^{\mu}$. If this condition is fulfilled, then Einstein equations imply that, with the form (6) of the stress-energy tensor, the relation

$$
\mathrm{NEC} \Longleftrightarrow \epsilon+p \geq 0,
$$

holds. This relation, whose violation implies the violation of all other energy conditions, is indeed assumed to hold in almost every situation of physical relevance. As we shall see, a bouncing Universe requires that it be violated in many circumstances.

Perturbations of the stress energy tensor (6) read

$$
\delta T_{0}^{0}=\delta \epsilon, \quad \delta T_{i}^{0}=\frac{\epsilon_{0}+p_{0}}{a} \delta u_{i}, \quad \delta T_{j}^{i}=-\delta p \delta_{j}^{u} .
$$

Here, and in the rest of this paper (unless otherwise stated), an index 0 on a quantity is meant to be the background, unperturbed value of this quantity. These perturbations are made gauge-invariant through the transformation $\delta \epsilon \rightarrow \delta \epsilon^{(\mathrm{gi})}=\delta \epsilon+\epsilon_{0}^{\prime}\left(B-E^{\prime}\right), \delta p \rightarrow \delta p^{(\mathrm{gi})}=$ $\delta p+p_{0}^{\prime}\left(B-E^{\prime}\right)$, and $\delta u_{i} \rightarrow \delta u_{i}^{(\mathrm{gi})}=\delta u_{i}+a\left(B-E^{\prime}\right)_{; i}$. Einstein equations then read, in terms of the Bardeen potential

$$
\begin{gathered}
\nabla^{2} \Phi-3 \mathcal{H} \Phi^{\prime}-3\left(\mathcal{H}^{2}-\mathcal{K}\right) \Phi=4 \pi G a^{2} \delta \epsilon^{(\mathrm{gi})}, \\
(a \Phi)_{; i}^{\prime}=4 \pi G a^{2}\left(\epsilon_{0}+p_{0}\right) \delta u_{i}^{(\mathrm{gi})}, \\
\Phi^{\prime \prime}+3 \mathcal{H} \Phi^{\prime}+\left(2 \mathcal{H}^{\prime}+\mathcal{H}^{2}-\mathcal{K}\right) \Phi=4 \pi G a^{2} \delta p^{(\mathrm{gi})},
\end{gathered}
$$

where the background equations $\left(\mathcal{H} \equiv a^{\prime} / a\right)$

$$
\begin{gathered}
\mathcal{H}^{2}+\mathcal{K}=\frac{8}{3} \pi G a^{2} \epsilon_{0}, \\
\frac{2}{3} \beta-\mathcal{H}^{2}-\mathcal{K}=\frac{8}{3} \pi G a^{2} p_{0},
\end{gathered}
$$

have been taken into account. In Eq. (13), we have used the variable $\beta$ defined as

$$
\beta \equiv \mathcal{H}^{2}-\mathcal{H}^{\prime}+\mathcal{K},
$$

which we will use intensively later on. It can be remarked, at this stage, that Eqs. (12) and (13) imply

$$
\epsilon_{0}+p_{0}=\frac{\beta}{4 \pi G a^{2}},
$$

so that a violation of the Null Energy Condition Eq. (7) will be equivalent to having $\beta<0$.
In general, pressure perturbations can be separated into adiabatic and entropic perturbations through

$$
\delta p=\left(\frac{\partial p}{\partial \epsilon}\right)_{S} \delta \epsilon+\left(\frac{\partial p}{\partial S}\right)_{\epsilon} \delta S=\Upsilon \delta \epsilon+\tau \delta S,
$$

with $\delta S$ the entropy perturbation. In many situations, $\Upsilon$ is interpreted as the squared sound velocity of the fluid and denoted $c_{s}^{2}$. However, for the fluids which appear in the examples below, this variable cannot be interpreted as such. Already in plasma physics there are situations where $\Upsilon^{1 / 2}$ is not the group velocity of the fluid (see Ref. [16], page 406, for a discussion on this point, and references therein). That is why we shall avoid calling $\sqrt{\Upsilon}$ the sound velocity in what follows.

Using Eq. (16), and plugging Eq. (11) into (9), one gets

$$
\begin{gathered}
\Phi^{\prime \prime}+3 \mathcal{H}(1+\Upsilon) \Phi^{\prime}-\Upsilon \nabla^{2} \Phi \\
+\left[2 \mathcal{H}^{\prime}+(1+3 \Upsilon)\left(\mathcal{H}^{2}-\mathcal{K}\right)\right] \Phi=4 \pi G a^{2} \tau \delta S,
\end{gathered}
$$

which becomes an homogeneous equation in the case of adiabatic perturbations $\delta S=0$ with which we shall be concerned from now on (see however the discussion in Sec. VB]).

We shall need, in what follows, two more pieces of information. It can be seen, by combining Eqs. (12), (13) and (16) that the following useful identity for $\Upsilon$

$$
\Upsilon=\frac{p_{0}^{\prime}}{\epsilon_{0}^{\prime}}=-\frac{1}{3}\left(1+\frac{\beta^{\prime}}{\mathcal{H} \beta}\right),
$$

holds. Moreover, it is clear that perturbation theory can only make sense if the perturbed Einstein tensor remains a small quantity. It turns out that Einstein equations do not involve anything but the Bardeen potentials. This stems from the fact that the gauge-invariant part of the Einstein tensor $\delta G_{\mu}^{(\mathrm{gi}) \nu}$ [the left-hand side of Eqs. (9) to (11)] only depends on $\Phi$ and $\Psi$ [20]. Satisfying the general requirements for perturbative analysis thus demands that not only the Bardeen potential, as discussed above, but also its first and second derivative in time, in order to remain a perturbation, should be regular and wellbehaved for all times. As we shall see later, this is not always a trivial requirement.

Hydrodynamical perturbations can be shown to originate from a single gauge-invariant variable $v(\eta, \mathbf{x})$, whose dynamics is derivable from the simple action

$$
S=\frac{1}{2} \int \sqrt{\gamma} \mathrm{d}^{4} x\left(v^{\prime 2}-\Upsilon \gamma^{i j} v_{, i} v_{, j}+\frac{z^{\prime \prime}}{z} v^{2}\right),
$$

where $\gamma$ is the determinant of the 3-metric $\gamma_{i j}$ (with inverse $\gamma^{i j}$ ), and the background function $z(\eta)$ is

$$
z^{2} \equiv \frac{a^{2}|\beta|}{\mathcal{H}^{2}|\Upsilon|},
$$

with $\beta$ defined in Eq. (14). The variable $v$ is formed with the fluid velocity perturbation potential and the Bardeen potential, its explicit form being here however irrelevant. 
Eq. (19) is in the correct form for a straightforward canonical quantization of the field $v$. There exists a connection between $v$ and the Bardeen potential which we will use below and that we therefore reproduce here. We first expand $\Phi$ on a basis of eigenfunctions of the LaplaceBeltrami operator (here, $k$ is a generic eigenvalue of this operator, which, depending on the curvature $\mathcal{K}$, can be continuous or discrete),

$$
\Phi=\sqrt{\frac{3}{4}} \ell \frac{\beta^{1 / 2}}{a} \int \mathrm{d} k\left(\psi_{k}(\mathbf{x}) u_{k}^{*}(\eta) a_{k}+\text { h.c. }\right),
$$

where $\ell \equiv \sqrt{8 \pi G / 3}$ is the Planck length, and $\psi_{k}(\mathbf{x})$ satisfies

$$
\left(\nabla^{2}+k^{2}\right) \psi_{k}(\mathbf{x})=0 .
$$

Implementing the usual commutation relations

$$
\left[a_{k}, a_{k^{\prime}}^{\dagger}\right]=\delta_{k k^{\prime}}
$$

(the $\delta$ here being a Dirac distribution or a Kronecker symbol depending on whether the space is flat or open, or closed, respectively) yields the time evolution equation for the wavelength-dependent modes of the Bardeen potential

$$
u_{k}^{\prime \prime}(\eta)+\left(\Upsilon k^{2}-\frac{\Theta^{\prime \prime}}{\Theta}\right) u_{k}(\eta)=0, \quad \Theta \equiv \frac{1}{\sqrt{|\Upsilon|} z}
$$

An expansion similar to Eq. (21) can be done for the field $v$. It turns out that its modes $v_{k}(\eta)$ satisfy

$$
v_{k}^{\prime \prime}(\eta)+\left(\Upsilon k^{2}-\frac{z^{\prime \prime}}{z}\right) v_{k}(\eta)=0
$$

and they are related to the Bardeen operator through the relation $\mathrm{H}$

$$
\Phi_{k}(\eta) \propto \frac{\sqrt{|\beta|}}{|\Upsilon|} \frac{z}{a}\left(\frac{v_{k}}{z}\right)^{\prime}
$$

A last interesting point concerning this field is that the eigenmodes are expected to be normalized as

$$
v_{k}^{\prime}(\eta) v^{*}(\eta)-v_{k}^{* \prime}(\eta) v_{k}(\eta)=2 i
$$

for all times $\eta$.

The process of generating primordial fluctuations then goes on by requiring the Universe to be empty at some early time, i.e. to be in the vacuum state $|0\rangle$ for which $a_{k}|0\rangle=0$ for all wavenumber $k$. The evolution of $v$ through Eq. (25) then necessarily changes the state and dynamically produces particles that are subsequently interpreted as primordial perturbations.

The background equations presented in this section will now be used for the forthcoming analysis of the bouncing background, to which we now turn.

\footnotetext{
${ }^{\ddagger}$ When $\beta$ or $\Upsilon$ change sign, it is necessary to calculate $\Phi$ directly by means of Eq. (17).
}

\section{GENERAL BOUNCING BEHAVIOR}

In this section we will enumerate some properties of a background spacetime with a bounce, and calculate some relevant quantities around the bounce which will be important for the calculations of the perturbations. We shall be mostly concerned in what follows by actual bounces, but the pseudo-bounce situation is also included in our description.

Our sole assumption on the background is that, near the last bounce before the standard model evolution took place, $a(\eta)$ can be written as follows

$$
a=a_{0}+b \eta^{2 n}+d \eta^{2 n+1}+e \eta^{2 n+2},
$$

where the integer $n$ satisfies $n \geq 1$. It means that $a(\eta)$ must be at least $\mathrm{C}^{2 n+2}$ near the bounce. In order that Eq. (28) indeed represents a bounce, the otherwise arbitrary parameter $b>0$. Our results are independent on what happens before this last bounce (other bounces, a $\mathrm{PBB}$ evolution, or even a singularity). In the case where $\epsilon_{0}=\epsilon_{0}(a)$, which is possible if and only if $a(\eta)$ is even, the presence of a bounce implies that the model is free of singularities. This is the case of models of Refs. [3, 10].

For this model to be realistic, we require $a_{0}$ to be less (or even much less) than $a_{n} \sim 10^{18} \mathrm{~cm}$, where $a_{n}$ is the value of the scale factor in the beginning of nucleosynthesis.

The function $\mathcal{H}(\eta)$ coming from Eq. (28) reads

$$
\begin{aligned}
\mathcal{H}= & \frac{1}{a_{0}^{2}}\left[2 n b a_{0} \eta^{2 n-1}+(2 n+1) a_{0} d \eta^{2 n}\right. \\
& \left.+(2 n+2) e a_{0} \eta^{2 n+1}-2 n b^{2} \eta^{4 n-1}\right],
\end{aligned}
$$

while $\beta(\eta)$ is

$$
\begin{aligned}
\beta= & \frac{1}{a_{0}^{2}}\left[\mathcal{K} a_{0}^{2}-a_{0} 2 n(2 n+1) d \eta^{2 n-1}-a_{0} 2 n(2 n-1) b \eta^{2 n-2}\right. \\
& \left.-a_{0}(2 n+2)(2 n+1) e \eta^{2 n}+2 n(6 n-1) b^{2} \eta^{4 n-2}\right] . \quad(30)
\end{aligned}
$$

From these functions we can calculate the coefficient $\Upsilon$ given in Eq. (18), yielding :

$$
\begin{aligned}
\Upsilon= & -\frac{a_{0}}{6 n b}\left\{\frac{-a_{0} 2 n(2 n-1)(2 n-2) b}{\eta^{2}}\right. \\
& +2[\mathcal{K} b-(2 n+2)(2 n+1) e] a_{0} n \\
& -\frac{2 a_{0} n\left(4 n^{2}-1\right) d}{\eta} \\
& \left.+16 b^{2} n^{2}(2 n-1) \eta^{2 n-2}+\ldots\right\} \\
& \left(\mathcal{K} a_{0}^{2}-a_{0} 2 n(2 n-1) b \eta^{2 n-2}+\ldots\right)^{-1} .
\end{aligned}
$$

We will now evaluate the behavior of the background functions $\mathcal{H}, \beta, \Upsilon$ and $z$ near the bounce for the possible values the free parameters can assume.

\footnotetext{
${ }^{\S}$ Or half-integer in the case of a pseudo-bounce.
} 
1. $\underline{n>1 \text { and } \mathcal{K} \neq 0 \text {. }}$

In this case, we find the following behaviors

$$
\begin{aligned}
\Upsilon & =\frac{(2 n-1)(2 n-2)}{3 \mathcal{K} \eta^{2}}, \\
\mathcal{H} & =\frac{2 n b}{a_{0}} \eta^{2 n-1}, \\
\beta & =\mathcal{K} \\
z & \propto \frac{1}{\eta^{2 n-2}}
\end{aligned}
$$

so that it turns out to be possible to have a bounce without violation of the NEC only if $\mathcal{K}=1$. Note that this case, as well as the following one, can accommodate a pseudo-bounce (where $2 n$ is an odd number strictly greater than 1 ).

2. $\underline{n>1 \text { and } \mathcal{K}=0 \text {. }}$

For the special case of a flat background, the various quantities needed to describe perturbations are modified as

$$
\begin{aligned}
\Upsilon & =-\frac{a_{0}(2 n-2)}{6 b n \eta^{2 n}}, \\
\mathcal{H} & =\frac{2 n b}{a_{0}} \eta^{2 n-1} \\
\beta & =-\frac{b}{a_{0}} 2 n(2 n-1) \eta^{2 n-2}, \\
z & \propto \text { const. }
\end{aligned}
$$

Here there is always violation of the NEC as $\beta \leq 0$ at least in some open neighborhood of the bounce.

3. $n=1, d \neq 0, \forall \mathcal{K}$.

This is the case where the second derivative of $a(\eta)$ is non vanishing and $a(\eta)$ is not even. The relevant quantities are

$$
\begin{aligned}
& \Upsilon=-\frac{a_{0} d}{b\left(2 b-\mathcal{K} a_{0}\right) \eta}, \\
& \mathcal{H}=\frac{2 b}{a_{0}} \eta, \\
& \beta=\mathcal{K}-\frac{2 b}{a_{0}}, \\
& z \propto \frac{1}{\sqrt{\eta}} .
\end{aligned}
$$

In this case, we can also have a bounce without violation of the NEC if $\mathcal{K}=1$ and $2 b / a_{0} \leq 1$. However, this condition is not physically plausible, as it is argued in the Appendix.

4. $n=1, \forall \mathcal{K}$, and $d=0$.

Finally, for the case that can represent an even $a(\eta)$, we get

$$
\begin{aligned}
\Upsilon & =\frac{8 b^{2}+(\mathcal{K} b-12 e) a_{0}}{3 b\left(2 b-\mathcal{K} a_{0}\right)}, \\
\mathcal{H} & =\frac{2 b}{a_{0}} \eta, \\
\beta & =\mathcal{K}-\frac{2 b}{a_{0}}, \\
z & \propto \frac{1}{\eta} .
\end{aligned}
$$

Here again, we can have a bounce without violation of the NEC if $\mathcal{K}=1$ and $2 b / a_{0}<1$.

This case corresponds to models of Refs. [3, 10].

Note that there are exceptional cases when $\mathcal{K}=1$ and $2 b=a_{0}$ in cases 3 and 4 , and when $8 b^{2}+(\mathcal{K} b-12 e) a_{0}=0$ in case 4 . For case 3 with $\mathcal{K}=1$ and $2 b=a_{0}$, it can be shown that unbounded growth occurs in the bounce, but we will not exhibit these calculations below because, as we have already mentioned, $\mathcal{K}=1$ and $2 b=a_{0}$ are not together plausible in realistic models. For case 4, we will show in the next section that the crucial instant of instability is not in the bounce itself, but when the Universe makes the transition from the region where the NEC is violated to the region where it is not. The occurrence of this transition is independent on the value of $8 b^{2}+(\mathcal{K} b-12 e) a_{0}$.

\section{NON-LINEAR COLLAPSE OF NON SINGULAR MODELS}

Having set the general conditions under which a bounce is expected to occur in the early Universe, we now apply the cosmological perturbation theory recalled in Sec. II to such a background. In particular, we shall make extensive use of Eqs. (117) and (24 26) to derive the leading terms in the behavior of the gauge-invariant Bardeen perturbation potential. Let us turn back to the time the bounce is supposed to have taken place, and consider the various situations for the free parameters already discussed above.

\section{A. The bounce}

There are four distinct cases near the bounce which deserve to be examined.

1. $n>1$ and $\mathcal{K} \neq 0$.

The first case is $n>1$ with non vanishing curvature $\mathcal{K}= \pm 1$. Eqs. (32) to (35) transform Eq. (25) into

$$
v_{k}^{\prime \prime}+\frac{(2 n-1)(2 n-2)}{\eta^{2}}\left(\frac{k^{2}}{3 \mathcal{K}}-1\right) v_{k}=0,
$$


whose solution is $v=v_{+} \eta^{p_{+}}+v_{-} \eta^{p_{-}}\left(v_{+}(k)\right.$ and $v_{-}(k)$ being two integration constants), with $2 p_{ \pm} \equiv$ $1 \pm \sqrt{1-4(2 n-1)(2 n-2)\left(\frac{k^{2}}{3 \mathcal{K}}-1\right)}$. For negative curvature space, $\mathcal{K}=-1$, one has $p_{-}<0$, for whatever value of $k$, while for $\mathcal{K}=1, p_{-}<0$ provided $k^{2}<3$. Note that $\forall k,\left(p_{-}-1\right)$ has a negative real part in both spaces $\mathcal{K}= \pm 1$.

Inserting this form into Eq. (26) yields, for the Bardeen potential, $\Phi_{k} \propto \eta^{p_{-}}$, i.e. also a divergent quantity for $p_{-}<0$. It can be seen from the previous considerations that, irrespective of the value of the wavenumber $k$, there will be a divergent quantity either in the metric perturbation directly for $\mathcal{K}=-1$, or in its derivative $\left(\propto \eta^{p_{-}-1}\right)$ for $\mathcal{K}=1$.

\section{2. $n>1$ and $\mathcal{K}=0$.}

The second case, similar to the first $(n>1)$ but for flat spatial sections $(\mathcal{K}=0)$, has $z=$ const. In order to calculate, the "pump" term, we need to obtain $z$ in the next to leading order of approximation. However, making use of the expansion formula for $v_{k}$

$$
\begin{aligned}
v_{k}= & z\left[A_{1}+A_{2} \int_{\eta_{0}}^{\eta} \frac{\mathrm{d} \hat{\eta}}{\hat{z}^{2}}\right. \\
& -k^{2} \int_{\eta_{0}}^{\eta} \frac{\mathrm{d} \hat{\eta}}{\hat{z}^{2}} \int_{\eta_{0}}^{\hat{\eta}} \mathrm{d} \tilde{\eta} \widetilde{\Upsilon} \tilde{z}^{2}\left(A_{1}+A_{2} \int_{\eta_{0}}^{\tilde{\eta}} \frac{\mathrm{d} \tilde{\tilde{\eta}}}{\tilde{\tilde{z}}^{2}}+\cdots\right],
\end{aligned}
$$

one can verify that $v_{k}$ contains a term proportional $k^{2} / \eta^{2 n-2}$, which diverges in the bounce.

In order to calculate what happens to the Bardeen potential $\Phi_{k}$, it is easier to investigate directly the Bardeen equation (17) when these values of the parameters are assumed, and one finds

$$
\Phi_{k}^{\prime \prime}+\frac{2-2 n}{\eta} \Phi_{k}^{\prime}-\frac{a_{0}(n-1) k^{2}}{3 b n \eta^{2 n}} \Phi_{k}=0,
$$

whose solution, setting

$$
\alpha \equiv k \sqrt{a_{0} /[3 n b(n-1)]} \eta^{1-n},
$$

is given by (see Eq. (8.491/3) in Ref. [22])

$$
\Phi_{k}=\eta^{(2 n-1) / 2}\left\{\Phi_{+} I_{\frac{2 n-1}{2 n-2}}(\alpha)+\Phi_{-} K_{-\frac{2 n-1}{2 n-2}}(\alpha)\right\},
$$

with two arbitrary constants of integration (depending on $k) \Phi_{+}$and $\Phi_{-}$. In this paper the symbols $J, N$ and $I, K$ stands for pairs of linearly independent solutions of the Bessel equation, Bessel and modified Bessel functions of first and second kind. As, by assumption, $n>1$, we find that the leading order behavior of the Bardeen potential is [Ref. 22], Eqs.(8.451/3-4)]

$$
\Phi_{k} \sim \eta^{(3 n-2) / 2} \mathrm{e}^{|\alpha|},
$$

namely, this perturbation exhibits a singular behavior near the bounce $(|\alpha| \rightarrow \infty)$.

3. $n=1, d \neq 0, \forall \mathcal{K}$.

This is the most general case, where $a(\eta)$ is not even and its second derivative is not null. Eqs. (44 47) when inserted into Eq. (25), yield

$$
v_{k}^{\prime \prime}+\left(\frac{\lambda(k)}{\eta}-\frac{3}{4 \eta^{2}}\right) v_{k}=0
$$

The explicit form of $\lambda$ is not important. The solution of this equation reads [Ref. [22], Eqs.(8.404/12)]

$$
v_{k}=\sqrt{\eta}\left\{v_{+} J_{2}(2 \sqrt{\lambda \eta})+v_{-} N_{2}(2 \sqrt{\lambda \eta})\right\},
$$

where $v_{+}, v_{-}$are arbitrary constants depending on $k$. The leading order behavior of the divergent term is $v_{k} \propto 1 / \sqrt{\eta}$. The Bardeen potential can be obtained from Eq. (26), or directly from Eq. (17) (with $\delta S=0$ ), yielding, near $\eta=0$,

$$
\Phi_{k} \propto \sqrt{\eta}\left\{v_{+} J_{1}(2 \sqrt{\lambda \eta})+v_{-} N_{1}(2 \sqrt{\lambda \eta})\right\},
$$

which is convergent at $\eta=0$. However, the first derivative of $\Phi_{k}$ has a divergent term,

$$
\Phi_{k}^{\prime} \propto \sqrt{\eta} N_{0}(2 \sqrt{\lambda \eta}),
$$

which behaves as $\ln \eta$ near $\eta=0$, and the second derivative is

$$
\Phi_{k}^{\prime \prime} \propto \frac{1}{\eta},
$$

yielding unbounded growth of the perturbed Einstein tensor (curvature) near the bounce.

4. $n=1, \forall \mathcal{K}$, and $d=0$.

This is the case which contains models from Refs. [3, 10], namely, that for which the first term in the scale factor expansion is quadratic $(n=1)$, for whatever value of the spatial curvature $\mathcal{K}$, and which can accommodate the case for which $a(\eta)$ is even.

Eqs. (40 43), inserted into Eq. (25), yield

$$
v_{k}^{\prime \prime}-\frac{2}{\eta^{2}} v_{k}=0,
$$

yielding the divergent mode $v_{k} \propto 1 / \eta$. For the Bardeen potential, we can use Eq. (24), or directly 
Eq. (17) (with $\delta S=0$ ), to obtain that, near $\eta=0$, it behaves as

$$
\Phi_{k} \propto \cos (\alpha(k, \mathcal{K}) \eta)
$$

which is regular up to second order derivatives independently on the form of the function $\alpha(k, \mathcal{K})$.

This is a case where there is no divergence in the bounce itself. However, as shown in the Appendix, near the bounce of a realistic Universe of this type, $\beta<0$, even when $\mathcal{K}=1$. As the Universe must have reached a region in which the NEC must be satisfied at later times, there must exist an instant $\eta_{0}$ at which the function $\beta$ vanishes. As it turns out, this is a critical point also from the point of view of perturbation theory.

\section{B. NEC transition}

In what follows, we now concentrate on the point where $\beta=0$, so that we shift the origin of time : for now on, $\eta_{0}=0$ for $\beta_{0}$, and we denote by an index 0 quantities evaluated at this point.

We now assume that the scale factor around $\eta=0$ is, again, derivable at least up to third order, so that the following expansion

$$
a(\eta)=a_{0}\left[1+\mathcal{H}_{0} \eta+\frac{1}{2}\left(2 \mathcal{H}_{0}^{2}+\mathcal{K}\right) \eta^{2}+\frac{1}{3 !} a_{3} \eta^{3}+\cdots\right]
$$

holds. In this relation, $a_{3} \equiv a^{\prime \prime \prime}(0) / a_{0}$, and use has been made of

$$
\frac{a^{\prime \prime}(0)}{a_{0}}=2 \mathcal{H}_{0}^{2}+\mathcal{K},
$$

which is a simple rewriting of $\beta_{0}=0$.

Using the expansion (60), we find that

$$
\begin{aligned}
\mathcal{H}= & \mathcal{H}_{0}+\eta\left(\mathcal{H}_{0}^{2}+\mathcal{K}\right) \\
& +\eta^{2}\left(\frac{a_{3}}{2}-2 \mathcal{H}_{0}^{3}-\frac{3}{2} \mathcal{K} \mathcal{H}_{0}\right)+\mathcal{O}\left(\eta^{3}\right),
\end{aligned}
$$

and

$$
\beta=\left(6 \mathcal{H}_{0}^{3}+5 \mathcal{K} \mathcal{H}_{0}-a_{3}\right) \eta+\mathcal{O}\left(\eta^{2}\right) .
$$

As shown in the Appendix, $\beta_{0}^{\prime} \neq 0$ for realistic models and hence $6 \mathcal{H}_{0}^{3}+5 \mathcal{K} \mathcal{H}_{0}-a_{3} \neq 0$. As a result,

$$
\Upsilon=-\frac{1}{3 \mathcal{H}_{0} \eta}+\mathcal{O}\left(\eta^{0}\right),
$$

which transforms Eq. (17) near $\eta=0$ into

$$
\frac{d^{2} \Phi_{k}}{d \eta^{2}}+\frac{1}{\eta} \frac{d \Phi_{k}}{d \eta}+\left(\frac{\mathcal{K}}{\mathcal{H}_{0}}-\mathcal{H}_{0}-\frac{k^{2}}{3 \mathcal{H}_{0}}\right) \frac{\Phi_{k}}{\eta}=0,
$$

whose solution reads ( [22], Eq.(8.494/5))

$$
\begin{aligned}
\Phi_{k}= & \Phi_{I} \eta I_{2}\left[2 \sqrt{\left(-\frac{\mathcal{K}}{\mathcal{H}_{0}}+\mathcal{H}_{0}+\frac{k^{2}}{3 \mathcal{H}_{0}}\right) \eta}\right] \\
& +\Phi_{K} \eta K_{2}\left[2 \sqrt{\left(-\frac{\mathcal{K}}{\mathcal{H}_{0}}+\mathcal{H}_{0}+\frac{k^{2}}{3 \mathcal{H}_{0}}\right)} \eta\right.
\end{aligned},
$$

where $\Phi_{I}$ and $\Phi_{K}$ are arbitrary constants depending on $k$, while $I_{2}$ and $K_{2}$ are ordinary Bessel functions. For realistic models, $\mathcal{H}_{0} \gg 1$. To lowest order, the Bardeen potential is then found as

$$
\Phi_{k} \sim \text { const. }+\mathcal{O}(\eta)
$$

which would seem to imply a safe perturbative expansion. However, its second derivative with respect to conformal time exhibits, irrespective of the value of $k$, the mildly diverging behavior

$$
\Phi_{k}^{\prime \prime} \sim \ln \eta
$$

so that, by virtue of Eqs. (9) - (11), we are forced to conclude that even this perturbative expansion generates unbounded growth.

\section{DISCUSSION}

The various cases that have been investigated here represent an exhaustive list of all the possibilities that satisfy the requirements of $i$ ) having a non-strictly monotonic behavior for the scale factor at some instant of time, ii) that this instant of time be in our past light cone, and that iii) therefore, the Universe in which the bounce took place is ours, in the sense that nucleosynthesis as we know it occurred. In all these cases, a divergent behavior for the cosmological hydrodynamical perturbations have been found, which are responsible for an observationally excluded non-linear growth of primordial inhomogeneities. We would like to discuss three points regarding these results.

$$
\text { A. } \Upsilon \rightarrow \infty \text {. }
$$

The first important point we should like to emphasize concerns the divergent behavior for $\Upsilon$, which could be expected to be responsible, right from the outset, for the divergence of the perturbations. That this is not the case can be seen on the following example, based on the last case of the previous section. If $\beta^{\prime}=0$, i.e., if $\beta \propto$ $\eta^{2 n+1}$ with $n \geq 1$, when $\beta=0$, then, to leading order, $\Upsilon$ behaves as $\Upsilon \sim-\frac{2 n+1}{3 \mathcal{H}_{0} \eta}$, and Eq. (65) is transformed in such a way that the Bardeen potential now reads 


$$
\begin{aligned}
\Phi_{k}= & \Phi_{I} \eta^{n+1} I_{2 n+2}\left[2 \sqrt{\left(-\frac{\mathcal{K}}{\mathcal{H}_{0}}+\mathcal{H}_{0}+\frac{k^{2}}{3 \mathcal{H}_{0}}\right) \eta}\right] \\
& +\Phi_{K} \eta^{n+1} K_{2 n+2}\left[2 \sqrt{\left(-\frac{\mathcal{K}}{\mathcal{H}_{0}}+\mathcal{H}_{0}+\frac{k^{2}}{3 \mathcal{H}_{0}}\right) \eta}\right],
\end{aligned}
$$

which has convergent derivatives up to order $2 n+2$. This is a case where, even in the point where $\Upsilon$ diverges, the curvature and all other relevant quantities converge. It happens because $\delta \epsilon$ goes to zero at this point. Hence, stable models with a divergent $\Upsilon$ at one point are indeed possible, as this simple example testifies. It is the combination of all the conditions i), ii) and iii), that gives rise to unbounded growth.

\section{B. Gauge invariance}

In each of the cases investigated above, we have found a growing mode for the Bardeen potential, which leads to divergences either in the metric perturbation or in the curvature. In view of recent discussions of similar modes found in the PBB case for instance [23], a natural question to ask then is whether they are actually physical. Here, we should like to argue that indeed, and contrary to their counterpart in $\mathrm{PBB}$, they cannot be "gaugeddown". In this sense, they represent real physical inhomogeneities, doomed to grow non-linear.

It is well-known that for wavelength larger than $\mathcal{H}^{-1}$, a residual gauge artifact might exist that can be tamed for $k \ll \mathcal{H}$ (for instance by going to an off-diagonal gauge in which higher order derivatives are involved [23]). However, the divergences we have obtained in the last section are present for all wavelengths. Furthermore, contrary to the PBB case, our divergences occurs on regular points of spacetime, where $\mathcal{H}$ is regular (even zero in the bounce, implying that all wavelengths are smaller then the Hubble radius at this time), and it is not possible to blame a bad behavior of $\mathcal{H}$ for their existence.

Let us see that explicitly. Assume one makes the calculations in the longitudinal gauge for which $B=E=0$ (note that this is not the case of the present paper). In this gauge, Eq. (3) simplifies to

$$
\mathrm{d} s^{2}=a^{2}(\eta)\left[(1+2 \phi) \mathrm{d} \eta^{2}-(1-2 \psi) \gamma_{i j} \mathrm{~d} x^{i} \mathrm{~d} x^{j}\right],
$$

where both $\psi$ and $\phi$ take the gauge-invariant value $\Phi$. We now assume that there exists a gauge transformation $x^{\alpha} \rightarrow \tilde{x}^{\alpha}=x^{\alpha}+\xi^{\alpha}(\mathbf{x}, \eta)$ that could cancel the divergence in the potential $\Phi_{k}$, leading to (in Fourier space, $\xi_{k}^{\alpha}(\eta)$ being the $\mathcal{K}$-dependent Fourier transform of the infinitesimal vector field $\left.\xi^{\alpha}\right) \tilde{\phi}_{k}=\Phi_{k}-\mathcal{H} \xi_{k}^{0}(\eta)-\xi_{k}^{0 \prime}(\eta)$, $\tilde{\psi}_{k}=\Phi_{k}+\mathcal{H} \xi_{k}^{0}, \tilde{B}_{k}=\xi_{k}^{0}-\xi_{k}^{\prime}$ and $\tilde{E}_{k}=-\xi_{k}$. The second of these relations reveals that since $\Phi_{k}$ is a divergent quantity at the physical point of space-time under consideration, the relevant transformation must be singular in order to produce a bounded value for $\psi_{k}$. This is to be contrasted with the $\mathrm{PBB}$ case for which, as $\eta_{P B B} \rightarrow 0$, $\mathcal{H}_{P B B} \rightarrow \eta^{-1}$ so that the gauge vector $\xi$ can indeed assume infinitesimal values while canceling the divergence. In our case, $\mathcal{H} \rightarrow \mathcal{H}_{0}$, a constant which, at the bounce, actually vanishes, thereby rendering the transformation even more singular. However we look at these perturbations, they do grow infinitely, leading to singularities, or at least to large (non-linear) inhomogeneities.

This can also be seen by using the gauge invariant curvature perturbation variable 24]

$$
\zeta=\Phi+\frac{\mathcal{H}^{2}+\mathcal{K}}{\mathcal{H} \beta}\left(\Phi^{\prime}+\mathcal{H} \Phi\right),
$$

which also exhibits divergences in the cases of interest here as can readily be checked. However, one should use this variable cautiously as the corresponding divergences may not be physical : in deriving this form for the threecurvature modes, it is explicitly assumed that the NEC is satisfied, so that for $\beta \rightarrow 0$, a fake divergence can occur. This is indeed what happens in the situation for which $\beta \propto \eta^{2 n+1}$ mentioned in Sec. $\mathrm{VA}:$ in this case, $\zeta \rightarrow \infty$ even though nothing particular happens in the theory at this point.

\section{Adiabatic and entropy perturbations}

The analysis that is presented here is restricted to purely adiabatic perturbations, which might be seen as too restrictive an hypothesis in view of the fact that at least two barotropic fluids are needed in a hydrodynamical description to yield a bounce. Bardeen equation (17) for the potential $\Phi$ can be read as an inhomogeneous equation with a source term proportional to the entropy perturbation, if the latter is independent of the former. In this situation, its solution is given by a particular solution of the full inhomogeneous equation, whatever it may be, plus the general solution of the homogeneous one, i.e. Eq (17) with $\delta S=0$, which is the equation we have been discussing throughout. As it was found that the adiabatic perturbations do exhibit a singular behavior near the bounce or at the time where the NEC is recovered, all the perturbations will exhibit such a behavior.

What if the entropy perturbation depends on the Bardeen potential ? In that case, it is useful to recall that an arbitrary perturbation can be decomposed over its possible modes (in the linear regime with which we are concerned), namely one adiabatic, and $N-1$ isocurvature modes for the case of $N$ constituents. All these modes may be well behaved, but one sees that with the decomposition 25

$$
\Phi=\Phi_{\text {adiabatic }}+\sum_{i=1}^{N-1} \Phi_{\text {isocurvature }}^{(i)}
$$


it is sufficient that the adiabatic mode we have been studying diverges to yield a cosmological catastrophe, unless the isocurvature modes somehow compensate exactly this divergence. Here, again, we return to recent observations of the CMB [7] according to which the dominant part of the primordial perturbations was in the adiabatic mode. Therefore, once again, the requirement that the bouncing Universe be ours implies a stringent constraint : in this case, it is that the adiabatic mode under scrutiny here is not negligible, and therefore cannot be compensated by an isocurvature mode.

\section{CONCLUSIONS}

The simplest way to envisage a cosmological model without an initial singularity is to think that the scale factor never shrunk to zero but reached a minimal value, preceded by a contraction phase. In fact, much of nonsingular cosmological models proposed in the literature present this bouncing behavior.

We have shown in the present paper that any bouncing solution whose matter content is described by hydrodynamical fluids is highly unstable, at least if they are to be smoothly joined with our observed Universe. In particular, applying to these solutions the now standard cosmological hydrodynamical perturbation theory, one finds that in all cases of interest, the Bardeen potential, which represents the typical scalar metric fluctuations, possesses divergent modes near the bounce that cannot be gauged away. For the quantum modes, these divergences appear because the so-called "pump" term $z^{\prime \prime} / z$ acts as an infinitely deep potential well : the corresponding $v$-wave then turns out to be unbounded for $\eta=0$, so that an infinite amount of particles are produced. This is similar to the divergent mode in ordinary inflationary scenarios, with one fundamental difference : in the usual scenarios, the divergence comes from the singular point $(a=0)$ of the metric, and can therefore safely be neglected, or altogether ignored, on the basis that physics cannot be described by any known theory at this point. Near a bounce, however, such an argument fails as one may construct perfectly consistent theories according to which Einstein gravity and hydrodynamics should hold at the bounce.

The divergent behavior which is obtained in all relevant cases actually means that any perturbation present at the time a bounce occurs would rapidly become nonlinear. In some cases, this unbounded growth happens in the bounce itself. If, in addition, the bounce implies a violation of the NEC, non linearities appear in at least one other situation : when the NEC is recovered. Hence, in many models, one have divergences in two or more moments of their history. As such a phenomenon happens for all wavelengths (the Hubble radius begin infinite near the bounce), one would then expect an ensemble of very strong inhomogeneities to be formed prior to any regular phase of the Universe.**

Note that this does not preclude the actual Universe to have a remote bounce. It is possible that after the inhomogeneities are formed, a small region of the Universe begins to inflate and eventually yields our Universe, much like in chaotic inflation, rendering the bounce spacelike separated from us (thereby departing from our hypothesis of physically observable bounce). The bounce in itself is very likely to be quite far from any observational verification. Its only interest, at the present time, is to reveal that it is theoretically possible to construct non singular cosmological models.

Summarizing, a Universe filled only with hydrodynamical fluids and with an observable bounce would already have been very highly inhomogeneous by the time nucleosynthesis initiates. This conclusion is, from the point of view of observational cosmology, inadmissible. This shows that one at least of the following statement is true :

i) No observable bounce ever took place in the Universe, or, stated differently, the Universe always expanded.

ii) If an observable bounce took place, then the dominant contribution in the stress-energy sourcing Einstein equations was not expressible in the form of a perfect fluid so that hydrodynamic cosmological perturbations do not make sense.

iii) Einstein equations are not valid in the time region where the Universe bounced.

Hence, in the framework of our hypothesis (4dimensional GR and hydrodynamical perturbations), we are therefore led to postulate a No-Bounce Conjecture according to which the opening question can be positively answered : from a pragmatic point of view, the Universe has always expanded.

\section{ACKNOWLEDGMENTS}

We would like to thank $\mathrm{CNPq}$ of Brazil for financial support. PP should like to acknowledge CBPF for hospitality during the time this work was being done. We also would like to thank N. Deruelle, J. Martin and the group of "Pequeno Seminário" for various enlightening discussions.

\footnotetext{
${ }^{* *}$ There is the very specific case where the initial conditions, for all wavelength $k$, are chosen such that the diverging mode is vanishing at all times, and if all the solutions of the inhomogeneous equation, when $\delta S \neq 0$, are regular. Such an extreme choice cannot, of course, be ruled out on purely mathematical grounds, but it seems fairly unlikely. In the models where the divergences appear more then once in their history, even mathematically this may not be possible.
} 


\section{APPENDIX : CONNECTING THE BOUNCE WITH THE RADIATION DOMINATED ERA OF THE STANDARD MODEL}

In this appendix we will prove that $\beta<0$ and $\beta^{\prime} \neq 0$ near the bounce when the conditions of case 4 are valid $\left(a(\eta)=a_{0}+b \eta^{2}+\ldots\right.$ in the neighborhood of the bounce).

A.

To prove that $\beta<0$, we will restrict ourselves to the single non trivial case $\mathcal{K}=1$, where $\beta=1-2 b / a_{0}$.

Considering conformal time ranging from a neighborhood of the last bounce until standard model evolution ( $a$ has the minimum value $a_{0}$ in this range), one can define without ambiguity the function

$$
f(\eta)=\sqrt{\frac{a^{2}(\eta)-a_{0}^{2}}{2 b a_{0}}},
$$

or the inverse relation

$$
a(\eta)=\sqrt{a_{0}^{2}+2 b a_{0} f^{2}(\eta)},
$$

where all initial conditions and physical parameters of the solution are contained in $b$ and $a_{0}$, which means that $f(\eta)$ is a pure a-dimensional mathematical function.

Near the bounce we obtain that $f(\eta)=\eta+O\left(\eta^{3}\right)$ (remember that this is the case $d=0$ ). Furthermore, any such model must be connected with the cosmological standard model before nucleosynthesis, which means that from $\eta=\eta_{n} \sim 10^{-11}$ to $\eta=\eta_{e q} \sim 10^{-4}$ (equal matter and radiation equilibrium) one should have

$$
a(\eta) \sim \bar{a} \sin (\eta) \sim \bar{a} \eta,
$$

where $\bar{a} \sim 10^{29} \mathrm{~cm}$. Substituting this expression in Eq. (73), and knowing that $a_{0}<a_{n} \sim 10^{18} \mathrm{~cm}$, we get

$$
f(\eta) \sim \frac{\bar{a}}{\sqrt{2 b a_{0}}} \eta .
$$

However, we know that around $\eta=0$ the function $f$ is exactly

$$
f(\eta)=\eta+\frac{f^{\prime \prime \prime}(0)}{6 !} \eta^{3}+\ldots
$$

If $2 b / a_{0}<1$ then $2 b a_{0}<a_{0}^{2}<a_{n}^{2}$ and $\bar{a} / \sqrt{2 b a_{0}}>10^{11}$. Knowing that Eq. (76) is valid already at $\eta \sim 10^{-11}$, the compatibility of Eqs. (76), (77) and $2 b / a_{0}<1$ is possible only if $f^{\prime \prime \prime}(0)$ and higher derivatives be a-dimensional huge number, which must be extremely fine tuned in order to yield the factor 1 multiplying the $\eta$ term in the above expansion. $\dagger$ Physicists believe that no reasonable dimensionless function $f(\eta)$ should contain such fine tuned very large a-dimensional numbers, so we conclude that $f(\eta)=\eta+\ldots$ for $\eta<\eta_{e q} \sim 10^{-4}$ 苗, and that $\bar{a}=\sqrt{2 b a_{0}} \sim 10^{29} \mathrm{~cm}$ which implies that $2 b / a_{0}>10^{22}$. Hence, $\beta=1-\frac{2 b}{a_{0}}$ is negative, and also in this case the $\mathrm{NEC}$ is violated.

B.

We will now prove that $\beta^{\prime} \neq 0$ around the point where $\beta$ changes sign. The change in sign of $\beta$ must occur before nucleosynthesis. Hence we can write

$$
a(\eta)=\sqrt{2 b a_{0} \eta^{2}+a_{0}^{2}} .
$$

Calculating $\beta$ given in Eq. (14) and equating it to zero we get the following quartic equation :

$\mathcal{K} 4 a_{0}^{2} b^{2} \eta^{4}+4 b a_{0}^{2}\left(\mathcal{K} a_{0}+2 b\right) \eta^{2}+a_{0}^{3}\left(\mathcal{K} a_{0}-2 b\right)=0$.

If $\beta^{\prime}=0$ when $\beta=0$, then $\beta^{\prime \prime}$ must also be zero. If it is not, then $\beta$ is not changing sign. Hence, $\beta$ must be of the form $\beta=A\left(\eta-\eta_{1}\right)\left(\eta-\eta_{0}\right)^{3}$ if $\beta^{\prime}=0$, where $\eta_{0}$ is some root of the quartic equation (79), $\eta_{1} \neq \eta_{0}$, and $A$ is a constant. But the polynomial form of Eq. (79) does not contain any cubic $\eta^{3}$ term so that $\beta$ cannot have three equal roots. Hence, we must conclude that $\beta^{\prime} \neq 0$.

[1] S. W. Hawking, G. F. R. Ellis, The large scale structure of space-time, Cambridge University Press (1973); R. M. Wald., General Relativity, Chicago University Press (1984). See also A. Borde, A. Vilenkin, Phys. Rev. D 56, 717 (1997) for a more recent discussion.

[2] E. W. Kolb, M. S. Turner, The Early Universe, AddisonWesley (1990).

[3] G. Murphy, Phys. Rev. D8, 4231 (1973); M. Novello and J. M. Salim, Phys. Rev. D20, 377 (1979); V. Melnikov and S. Orlov, Phys. Lett A70, 263 (1979); E. Elbaz, M. Novello, J. M. Salim and L. A. R. Oliveira, Int. J. of Mod. Phys. D1, 641 (1993).

\footnotetext{
${ }^{\dagger \dagger}$ One possible mathematical function which is compatible with these requirements is $f(\eta)=\left(1-10^{14}\right) \eta \exp \left(-10^{25} \eta^{2}\right)+$ $10^{14} \eta$. Note, however, the huge numbers present in this function, and the presence of the term $1-10^{14}$. When $\eta$ goes to zero, the difference of two numbers of order $10^{14}$ must be exactly 1 , an extreme fine tuning !

$\ddagger \ddagger$ One conclusion from these considerations is that the scale factor solution obtained in Ref. 10 is the most general physically reasonable one, up to matter domination, which can accommodate a bounce with our real Universe.
} 
[4] R. C. Tolman, Phys. Rev. 38, 1758 (1931).

[5] G. Veneziano, Phys. Lett. B 265, 287 (1991); M. Gasperini, G. Veneziano, Astropart. Phys. 1, 317 (1993); See also J. E. Lidsey, D. Wands, E. J. Copeland, Phys. Rep. 337, 343 (2000) and G. Veneziano, String Cosmology : the Pre Big Bang scenario, Les Houches summer school, Eds. P. Binétruy et al., Elsevier Science Publishers (2001);

[6] R. Brustein, R. Madden, Phys. Lett. B 410, 110 (1997); Phys. Rev. D 57, 712 (1998).

[7] C. Netterfield et al., A measurement by BOOMERANG of multiple peaks in the angular power spectrum of the cosmic microwave background, astro-ph/0104460 R. Stompor et al., Cosmological implications of the MAXIMA-I high resolution Cosmic Microwave Background anisotropy measurement, astro-ph/0105062 P. De Bernardis et al., Nature (London) 404, 955 (2000); S. Hanany et al., Astrophys. J. Lett. 545, 5 (2000); G.F. Smoot et al., Astrophys. J. 396, L1 (1992); C.L. Bennett et al., Astrophys. J. 464, L1 (1996).

[8] J. Khoury, B. A. Ovrut, P. J. Steinhardt, N. Turok, The ekpyrotic universe: Colliding branes and the origin of the hot big bang, hep-th/0103239; R. Kallosh, L. Kofman, A. Linde, Pyrotechnic universe, hep-th/0104073; J. Khoury, B. A. Ovrut, P. J. Steinhardt, N. Turok, A brief comment on 'The Pyrotechnic universe', hep-th/0105212; R. Y. Donagi, J. Khoury, B. A. Ovrut, P. J. Steinhardt, N. Turok, Visible branes with negative tension in heterotic M-theory, hep-th/0105199, R. Kallosh, L. Kofman, A. Linde, A. Tseytlin, BPS branes in cosmology, hep-th/0106241

[9] D. A. Easson, R. H. Brandenberger, Universe Generation from Black Hole Interiors, hep-th/0103019 (2001)

[10] J. Acacio de Barros, N. Pinto-Neto, M. A. Sagioro-Leal, Phys. Lett. A 241, 229 (1998).

[11] R. Colistete Jr., J. C. Fabris, N. Pinto-Neto, Phys. Rev. D 62, 083507 (2000).

[12] The Many-Worlds Interpretation of Quantum Mechanics, Eds. B. S. De Witt and N. Graham, Princeton University Press (1973)

[13] D. Bohm, Phys. Rev. 85, 166 (1952); D. Bohm, B. J. Hiley, P. N. Kaloyerou, Phys. Rep. 144, 249 (1987); P. R. Holland, The Quantum Theory of Motion, Cambridge University Press (1993).

[14] N. Pinto-Neto, R. Colistete Jr, Graceful exit from inflation using quantum cosmology, gr-qc/0106063.

[15] C. Molina-Paris, M. Visser, Phys. Lett. B 455, 90 (1999).

[16] M. S. Morris and K. S. Thorn, Am. J. of Phys. 56, 395 (1988).

[17] Ya. B. Zel'dovich and L. P. Pitaevsky, Commun. Math. Phys. 23, 185 (1971); L. A. Wu, H. J. Kimble, J. L. Hall and H. Wu, Phys. Rev. Let. 57, 2520 (1986); M. Visser, Phys. Lett. B 349, 443 (1995); C. Barceló and M. Visser, Nucl. Phys. B 584, 415 (2000).

[18] D. Solomons, P. Dunsby, G. Ellis, No bounce behaviour in Kantowski-Sachs Cosmologies, gr-qc/0103087 (2001).

[19] G. Veneziano, it in Les Houches, Session LXXI, The primordial Universe, P. Binétruy et al. Eds., EDP sciences \& Springer (2000).

[20] V. F. Mukhanov, H. A. Feldman, R. H. Brandenberger,
Phys. Rep. 215, 203 (1992).

[21] J. M. Bardeen, Phys. Rev. D 22, 1882 (1980).

[22] I. S. Gradshteyn, I. M. Ryshik, Table of Integrals, Series and Products, Academic Press, New-York (1980).

[23] R. Brustein et al., Phys. Rev. D 51, 6744 (1995).

[24] R. Brandenberger, R. Kahn, W. Press, Phys. Rev. D 28, 1809 (1983); D. Lyth, Phys. Rev. D 31, 1792 (1985).

[25] D. Langlois, A. Riazuelo, Phys. Rev. D 62, 043504 (2000). 